\title{
PROMOÇÃO DA SAÚDE NO CONTEXTO DA ESTRATÉGIA SAÚDE DA FAMÍLIA: CONCEPÇÕES E PRÁTICAS DA ENFERMEIRA
}

\author{
Health promotion in the context of family health strategy: conceptions and practices of \\ nurse \\ Promoción de la salud en el contexto de la estrategia de salud de la familia: concepciones \\ y prácticas de enfermería
}

Maria Glêdes Ibiapina Gurgel ${ }^{1}$
Patrícia Neyva da Costa Pinheiro ${ }^{4}$

\section{RESUMO}

As concepções que os profissionais têm da promoção da saúde são fundamentais à prática. 0 estudo objetivou conhecer as concepções sobre promoção da saúde e a interface da atuação da enfermeira na Saúde da Família. Pesquisa descritiva, exploratória, com abordagem qualitativa, realizada com oito enfermeiras dos Centros de Saúde da Família de Fortaleza-CE. Utilizou-se o grupo focal para coleta de dados. A análise foi realizada por meio das práticas discursivas, tendo como recurso os mapas de associação de ideias. As concepções sobre promoção da saúde, evidenciadas pelas enfermeiras, perpassam ora pelo modelo biomédico de prevenção das doenças, ora pelo de promoção, apregoada na Carta de Ottawa. Nas concepções do modelo tradicional, as ações estão restritas ao setor saúde com enfoque na cura e na prevenção das doenças. As concepções do modelo de promoção da saúde aproximam-se do conceito de qualidade de vida, com suporte nas ações interdisciplinares e intersetoriais.

Palavra-chave: Enfermagem. Promoção da Saúde. Atenção Primária à Saúde

\section{Abstract}

The conceptions that professionals have about health promotion are fundamental to the practice. The study aimed to know the conceptions about health promotion and the interface of nursing care in Family Health. Descriptive and exploratory research, with a qualitative approach, carried out with eight nurses from the Centers for Family Health of Fortaleza-CE. We used the focal group for data collection. The analysis was performed by means of discursive practices, having as resource maps of association of ideas. The conceptions of health promotion, as evidenced by nurses, now permeate the biomedical model of disease prevention, either by promotion, trumpeted in the Ottawa Charter. In conceptions of the traditional model, the actions are restricted to the health sector with a focus on healing and disease prevention. The conceptions of the model of health promotion approach the concept of quality of life, supporting the interdisciplinary and intersectoral actions.

Keywords: Nursing. Health Promotion. Primary Health Care.

\section{Resumen}

Las concepciones que los profesionales han tenido sobre la promoción de la salud son fundamentales para la práctica. El estudio objetivó conocer las concepciones sobre promoción de la salud con interface en la actuación de la enfermera en Salud de la Familia. Investigación descriptiva, exploratoria, cualitativa, realizada con ocho enfermeras del Centro de Salud de la Familia de Fortaleza-CE, por medio del grupo focal en la recogida de datos y análisis de las prácticas discursivas con los mapas de asociación de ideas. Las concepciones sobre la promoción de la salud, como lo demuestran las enfermeras, a veces son las del modelo biomédico de prevención de enfermedades, otras son de la promoción, pregonada en la Carta de Ottawa. En la primera, las acciones se limitan a la curación y prevención de enfermedades, pero en la segunda, han señalado el concepto de calidad de vida, centrándose en la acción interdisciplinaria e intersectorial.

Palabra clave: Enfermería. Promoción de la Salud. Atención Primaria de Salud

\footnotetext{
'Doutoranda em Enfermagem pela Universidade Federal do Ceará. Enfermeira do Hospital Distrital Gonzaga Mota de Messejana da Secretaria de Saúde de Fortaleza., Fortaleza-CE. Brasil. E-mail: gledesgurgel@yahoo.com.br;²Doutora em Enfermagem, Professora Associada da Universidade Federal do Ceará. Fortaleza -CE. Brasil. E-mail dalva@ufc.br;'²Doutora em Enfermagem, Professora Adjunta da Universidade Federal do Ceará. Fortaleza-CE. Brasil. E-mail: escolpaz@yahoo.com.br; ${ }^{4}$ Doutora em Enfermagem, Professora Adjunta da Universidade Federal do Ceará.Fortaleza-CE. Brasil. E-mail: neyva.pinheiro@yahoo.com.br; 5 Mestre em Enfermagem, Professora Assistente da Universidade Federal de Sergipe.Fortaleza-CE. Brasil. E-mail: ritamvrego@gmail.com; ${ }^{M}$ Mestranda em Enfermagem pela Universidade Federal do Ceará. Enfermeira da Vigilância Epidemiológica da Secretaria Executiva Regional VI. Secretaria de Saúde de Fortaleza. Fortaleza-CE. Brasil. E-mail: leonicepassos@hotmail.com
} 


\section{INTRODUÇÃO}

0 conceito de promoção da saúde evolui ao longo do tempo. Foi visto, inicialmente, por Wislow em 1920, Sigeriste em 1946, Leavell e Clark em 1965 e Paim e Teixeira em 1993 como um nível de prevenção, posteriormente entendido como atividades dirigidas à transformação do comportamento de risco. Nas duas visões, as orientações eram voltadas para evitar o surgimento de doenças específicas e os profissionais adotavam como estratégia a divulgação da informação e recomendações normativas de mudanças de hábitos. As ações e discursos preventivos eram de cunho educativo e centradas na responsabilidade do próprio indivíduo., ${ }^{1,2}$

Após a Conferência de Alma Ata em 1978 e as Conferências Internacionais de Promoção da Saúde: Ottawa 1986, Adelaide 1988, Sandsvall 1991, Jacarta 1997 e México 1999, esse conceito ampliou-se com a inclusão dos aspectos e fatores relacionados à qualidade de vida; atividades voltadas à coletividade e ao meio ambiente; estratégias mais integradas e intersetoriais; e participação da população, desde a formulação até a implantação das políticas. Presume a capacitação do indivíduo para participar ativamente do autocuidado, havendo ampliado a perspectiva de relacionar saúde com as condições de vida e elementos sociais, políticos, econômicos e ambientais. ${ }^{3,4}$

Objetivando acelerar o progresso de promoção da saúde, foi formulada uma aliança mundial em 1978 sob a liderança da Organização Mundial de Saúde, intitulada "Saúde para todos no ano 2000", com a finalidade de exortar as instituições públicas sociais, econômicas e educacionais, organizações não governamentais, movimentos sociais, entidades de classe e setor privado a concederem as necessárias prioridades à promoção da saúde. ${ }^{3}$

0 Brasil vem investindo na formulação e implementação de políticas de promoção da saúde, concentrando esforços na construção de um modelo de atenção que priorize ações de melhoria da qualidade de vida dos sujeitos e coletivos. A Política Nacional de Promoção da Saúde, Escolas Promotoras de Saúde, 0 Pacto em Defesa do SUS, 0 Pacto em Defesa da Vida e 0 Pacto de Gestão são iniciativas que propõem a ampliação e qualificação das ações de promoção da saúde nos serviços e na gestão do Sistema Único de Saúde. ${ }^{5}$

0 movimento em prol da saúde surgiu no Brasil com a reforma sanitária, que resultou no reconhecimento da saúde como um direito do cidadão e dever do Estado, expresso no artigo 196 da Constituição Federal, em 1988.

Em 1990, foi instituído o Sistema Único de Saúde (SUS), um novo paradigma, com princípios e diretrizes que nor teiam a reorientação de um sistema de saúde equânime e resolutivo. Os avanços na saúde tiveram impulsos significativos com a implantação do Programa Saúde da Família (PSF), como estratégia de reorientação do sistema de saúde, com o desafio de obter uma inversão do modelo biomédico de atenção e proporcionar maior acesso aos serviços e melhor interação e participação comunitária; com ações que transcendam os espaços institucionais de saúde no sentido de identificar, otimizar e potencializar os recursos disponíveis na comunidade para as ações de promoção da saúde. ${ }^{6,7,8}$

0 novo paradigma da promoção da saúde passa pela academia e pela formação dos profissionais de saúde, com foco nas mudanças de atitude e de ressignificação das concepções e práticas, voltadas para as necessidades do indivíduo como um ser holístico e para um trabalho em rede. A ESF é considerada epicentro da rede e demandada a complemantariedade da atenção para outros níveis, assegurando assim a equidade e a integralidade da atenção, ${ }^{6,7}$

As concepções que a enfermeira tem da promoção da saúde são fundamentais e norteiam a sua prática. 0 entendimento de promoção da saúde é uma corresponsabilidade dos componentes da equipe da Saúde da Família, pela influência na atuação, e vai além de aprimorar a escuta, fortalecer os vínculos, garantir o acesso às informações. ${ }^{6,7}$ São primordiais a intersetorialidade, as ações coletivas para a promoção, desenvolvimento de atitudes e habilidades, aumentando na coletividade 0 poder de decisão, negociação e acesso a atividades educativas e recreativas, bem como 0 autocuidado. 9,10

A Enfermagem é uma profissão comprometida com a saúde e qualidade de vida da pessoa, família e coletividade. 0 cuidar/cuidado de Enfermagem está centrado na lei do exercido e no Código de Ética de Enfermagem; e sua atuação compreende a promoção, prevenção, recuperação e reabilitação da saúde, com autonomia e em consonância com os preceitos legais éticos e as políticas públicas de saúde. ${ }^{11}$

Em face das transformações ocorrentes no entendimento do processo saúde-doença e sua natureza, a Enfermagem busca reformular sua prática, mediante a aproximação com esse novo paradigma de promoção da saúde. Em qualquer esfera de atenção, há a necessidade de superar os limites e as concepções do cuidar/cuidado de Enfermagem, caracterizado por um modelo biomédico, hospitalocêntrico, fragmentado, individual e curativo. ${ }^{12}$

Ante esse contexto, optou-se por conhecer as concepções de promoção da saúde e a interface na atuação da enfermeira da ESF.

\section{PERCURSO MÉTODOLÓGICO}

Esta é um pesquisa descritivo-exploratória, com abordagem qualitativa. Trata-se de um recorte da dissertação de mestrado sobre prevenção da gravidez na adolescência no contexto da ESF, defendida pela primeira autora, e teve como ambiente investigativo oito Centros de Saúde da Família (CSF), de Fortaleza-CE, por adotarem a Saúde da Família como estratégia de organização da atenção primária, contexto favorável ao desenvolvimento de atividades de promoção da saúde. 
A amostra constou de enfermeiras vinculadas à ESF de Fortaleza. Foram definidos dois critérios de inclusão: desenvolver ações de saúde ao adolescente e ter pelo menos um ano de atuação no C.S.F. no momento da pesquisa.

A coleta de dados ocorreu por meio do grupo focal. A pesquisa atendeu aos critérios éticos da Resolução 196/96. ${ }^{13}$ Foi aprovada no Comitê de Ética Universidade Federal do Ceará sobre o Protocolo $n^{\circ}$ 282/07.

As enfermeiras foram identificadas com a letra $E$, seguida de um número, que variou de 1 a 8, para preservar 0 anonimato.

No grupo focal, a escolha das participantes ocorreu por sorteio e compreendeu três etapas. A princípio, foi feito o diagnóstico do Município de Fortaleza, com a identificação dos 19 CSF e 35 enfermeiras que atuavam na atenção ao adolescente; em seguida, houve o sorteio dos CSF, sendo selecionadas uma enfermeira da SER II, III, IV, V e duas da SER le Vl.

No convite para participar da pesquisa, as enfermeiras foram informadas somente da temática central, objetivo da pesquisa, modalidade da técnica e o compromisso com a pontualidade. 0 grupo focal foi planejado para seis participantes, mas, por medida de segurança no caso de eventuais ausências inesperadas, foram convidadas oito enfermeiras.

As sessões dos dois grupos focais aconteceram na sede da Associação Brasileira de Enfermagem, Seção Ceará. Foram promovidas duas reuniões grupais com as mesmas participantes. Nas sessões, a duração média das discussões ficou ao redor de duas horas. Usou-se o gravador, que permitiu o suporte para a transcrição com reprodução fiel e na íntegra das falas.

A primeira autora moderou os dois encontros e contou com três assistentes - uma relatora e duas observadoras, todas enfermeiras com experiência em grupo focal, cuja condução ocorreu com roteiro contendo perguntas norteadoras, pautadas nas concepções de promoção da saúde.

Os indicadores foram analisados por meio das práticas discursivas e produção de sentido no cotidiano, tendo como recurso os mapas de associação de ideias. As práticas discursivas constituem uma técnica de análise definida como linguagem em ação, isto é, as maneiras com amparo nas quais as pessoas produzem sentidos e se posicionam em relações sociais cotidianas.

Os mapas de ideias constituem a apresentação das entrevistas em tabelas, onde as colunas estão definidas por temáticas, sempre respeitando os objetivos propostos. Iniciouse a formulação do mapa pela definição da categoria, que reflete, sobretudo, o objetivo da pesquisa. No primeiro momento, constituem as formas de visualização das dimensões teóricas e de organização do conteúdo, preservando a sequência das falas; na segunda ocasião, mantêm o diálogo intato apenas deslocando para a coluna previamente definida.
Na elaboração do mapa, é fundamental seguir os passos: 1) utiliza-se um processador de dados, do tipo Word for Windows; 2) digita-se toda a entrevista; 3) elabora-se um quadro com o número de colunas correspondente às categorias a serem utilizadas; e 4) usa-se as funções cortar e colar para transferir o conteúdo do texto para as colunas, respeitando as sequências do diálogo. ${ }^{14}$

\section{RESULTADOS E DISCUSSÃO}

A idade média das enfermeiras foi de 32 anos, o tempo de graduação apresentou-se inferior a dez anos; e, em média, as participantes tinham cinco anos de atuação na ESF.

Os resultados das discussões do grupo focal foram transcritos em respeito à sequência da anunciação. Buscou-se trabalhar 0 aspecto dialogal implícito na produção do sentido e o encadeamento das associações de ideias com a categoria e subcategoria previamente definidas, com esteio no objetivo, cuja análise ocorreu mediante aproximação do discurso das participantes com a categoria Concepção de promoção da saúde e subcategorias baseadas no modelo de prevenção da doença (tradicional) e modelo de promoção da saúde (atual).

\section{Concepções de promoção da saúde}

Ao conceituar a promoção da saúde no primeiro grupo focal, quatro enfermeiras pautaram seu conceito no modelo de promoção da saúde e quatro no modelo tradicional de prevenção das doenças, como destacam as narrativas:

Para mim promoção da saúde está muito ligado a prevenção da doença (E1).

Promover tem tudo a ver com atitudes, com orientações ou com ajuda que faz com que uma comunidade se mantém saudável dentro daquilo que ela vive(E3).

Nesses discursos, pode-se perceber que há uma associação da ideia da promoção da saúde com o modelo de Leavel e Clark, que utiliza o conceito de promoção da saúde com base na história natural e na prevenção de doenças, com três níveis: prevenção primária, secundária e terciária.

Nesse modelo, a promoção da saúde está limitada ao primeiro nível de prevenção, a prevenção primária, que compreende a promoção da saúde e a prevenção das doenças. A primeira diz respeito a moradia adequada, escolas, áreas de lazer, alimentação e educação em todos os níveis. A segunda compreende as ações de imunização, saúde ocupacional, higiene pessoal e do lar, proteção contra acidentes, aconselhamento genético e controle de vetores. ${ }^{2}$

0 modelo tradicional de prevenção das doenças tem como centro das atenções o indivíduo doente, cuja responsabilidade pela cura é de competência exclusiva do 


\section{Promoção da saúde no contexto da estratégia saúde da família}

profissional. Com essa concepção, uma enfermeira nos dois encontros manteve o mesmo contexto em suas narrativas:

Promoção da saúde, como o nome diz, é promover; é dar um sentido. É dar uma forma de se ter saúde, a gente promovendo, estar dando condição daquela comunidade ter saúde. Promover é dar condição de acontecer. Quando se dá condições de informação a estes adolescentes, estamos promovendo a sua saúde (E4).

A diferença entre os enfoques de promoção da saúde e prevenção das doenças engloba vários aspectos, que vão desde os princípios e conceitos de saúde até a execução das ações.

No enfoque da prevenção das doenças, o objetivo da atenção é evitar a doença ou enfermidade, ou seja, o indivíduo passa a ser o alvo da intervenção e é responsável pelo seu estado de saúde; atribui ao seu comportamento os fatores de risco para determinada doença. ${ }^{2}$ Nessa visão, o direcionamento das medidas de proteção é voltado a patologias específicas e impostas a grupos-alvo, denominados grupo de riscos. Nesse contexto, veio à tona a concepção preventivista e campanhista:

Acho que para haver promoção em relação à comunidade, a gente tem que conhecer aquela comunidade, as suas necessidades e bolar estratégias de ações da promoção da saúde dependendo da carência, da realidade de cada comunidade. Isso é feito, acho que para o lado da promoção saúde; são as campanhas que a gente faz, dia D de hanseníase, as campanhas de educação, hipertensão e diabetes para a detecção de casos precoce; está muito na prevenção também, mas promoção é conhecer a realidade daquela comunidade e trabalhar aquelas carências maiores (E8).

A promoção da saúde difere da prevenção de doenças em diversos aspectos, pois na primeira o modelo de intervenção é participativo, trabalha com a mobilização comunitária e tenta romper o individualismo. ${ }^{1}$ Suas estratégias são diversas, complementares e direcionadas para toda a população no seu contexto, com o objetivo de mudar a situação dos indivíduos e do meio ambiente. As estratégias definidas não devem ser contingentes, mas sistemáticas e inseridas em planejamento estratégico. Houve destaque da relevância do planejamento nas ações de promoção:

São ações que a gente pode desenvolver e alcançar um objetivo nas ações de saúde. Você está desenvolvendo ações com diferentes temas de forma específica, organizada, esquematizada, planejada. Com objetivos e metas, você consegue chegar à chamada promoção da saúde (E6).

É benéfico destacar a importância da articulação entre as ações de promoção nos níveis de atenção primário, secundário e terciário e atuação intersetorial no território, como espaço de permanentes relações sociais e de pactuação. Em relação a essas pactuações, emergiu a ideia de promoção articulada com o reconhecimento do indivíduo como sujeito do processo:

São ações, que a gente vai utilizar junto ao individuo e comunidade ou grupo, em que eles possam se perceber sujeitos ativos de como eles podem manter sua saúde (E5).

A promoção da saúde tem enfoque mais abrangente e busca o protagonismo para identificar e enfrentar os macrodeterminantes do processo saúde doença, de modo a transformá-los, favoravelmente, em direção à saúde. Esse enfoque investe no exercício da cidadania com o objetivo de modificar as condições de vida, para que sejam dignas e adequadas. ${ }^{1,7}$

No decorrer das narrativas dos dois grupos focais, percebe-se uma aproximação da ideia de promoção da saúde à maneira mais ampla, como estratégia mediadora entre pessoas e ambiente, visando a aumentar a participação dos sujeitos e da coletividade na modificação dos determinantes do processo saúde doença, como emprego, renda, educação, lazer e hábitos de vida saudáveis. ${ }^{7,9,15}$

A promoção desencadeia um processo para aumentar a saúde e o bem-estar, com estratégias de transformações das condições de vida, mediadas por atividades que estimulem as potencialidades pessoais e promovam a motivação. ${ }^{7,16}$ Essa aproximação foi destacada na fala:

A promoção da saúde é uma estratégia, capaz de estimular aquele a quem você dirige o cuidado. Todas as pessoas têm um potencial, e, quando você promove a saúde, você vai lá naquele potencial é como uma descarga elétrica. Você estimula esse potencial, e aquelas pessoas dão um salto de qualidade, por melhor que sejam, querendo elas podem, ser mais e melhor (E7).

Mesmo com os discursos buscando a aproximação da promoção da saúde com a feição mais ampla, é importante destacar o fato de que as enfermeiras pouco expressaram elementos indispensáveis à promoção da saúde, como o "empoderamento", a autonomia, o diálogo e a visão de mundo. ${ }^{16}$

A promoção da saúde demanda inúmeras estratégias de intervenção na realidade. 0 planejamento das ações deve 


\section{Promoção da saúde no contexto da estratégia saúde da família}

ser ancorado na vigilância à saúde e no reconhecimento da determinação social no processo saúde-doença, arrimado, também, na percepção das vulnerabilidades e nas implicações das condições de vida sobre as condições de saúde da população.

\section{CONCLUSÃO}

A concepção que o profissional de saúde tem de promoção da saúde influencia significativamente a sua prática e é efetivada por meio de suas ações. 0 conceito de promoção da saúde, mais amplo, como qualidade de vida, direciona a atuação da enfermeira de modo a desenvolver uma visão aprimorada do contexto socioeconômico e cultural da população, como também conhecer, compreender e considerar os determinantes e condicionantes como indicadores que ampliam ou reduzem as vulnerabilidades dos grupos populacionais. Esses aspectos possibilitam a aproximação dos saberes com prática na ESF, voltadas às perspectivas da promoção da saúde.

Nos discursos, as concepções sobre promoção da saúde, evidenciadas pelas enfermeiras, passam ora pelo modelo biomédico de prevenção das doenças, ora pelo de promoção, conforme apregoado pela Carta de Ottawa.

As ideias de promoção da saúde no primeiro grupo focal estavam mais voltadas ao modelo tradicional, ações restritas ao setor saúde, com foco na cura e na prevenção das doenças, e seu significado era atribuído ao primeiro nível de atenção. No segundo grupo, essas concepções foram ampliadas, aproximando de qualidade de vida o conceito de promoção da saúde, em que foram consideradas várias dimensões e contexto, incluindo também o enfoque em ações interdisciplinares e intersetoriais.

Após as discussões acerca das concepções de promoção da saúde, as enfermeiras articularam com suas práticas, percebendo que as ações de promoção da saúde são incipientes, mesmo com os esforços empreendidos.

0 planejamento das ações de promoção da saúde, elaborado em alguns centros de saúde, na maioria das vezes atende às demandas provenientes das secretarias executivas regionais e da Secretaria Municipal de Saúde e, raramente, realizam avaliação do trabalho.

As ações de promoção da saúde são trabalhadas de forma individual, na consulta de Enfermagem, e coletivamente nos grupos de adolescentes, gestantes, planejamento familiar, hipertensos e diabéticos. Acredita-se ser o grupo um espaço criativo, interativo e oportuno para tratar de vários aspectos da promoção da saúde.

A enfermeira desempenha relevante papel na equipe e deve promover ações interdisciplinares de educação em saúde que integrem família, escola e comunidade, despertando 0 interesse e conhecimento de suas potencialidades.

Nos relatos das concepções e experiências, as enfermeiras revelam aspectos para se (re)pensar estratégias de promoção da saúde pela equipe da ESF.
Reportamo-nos a algumas recomendações que, sobremaneira, auxiliarão na feitura de agendas que vislumbrem a promoção da saúde:

- Os centros de saúde da família devem realizar estudos para identificar nos adolescentes de sua área de abrangência fatores protetores e vulnerabilidades sociais, econômicas e culturais, e utilizar os indicadores de saúde como subsídios para o planejamento das ações;

- As enfermeiras da ESF devem estar em educação permanente, com vistas à ressignificar seus conceitos e sua prática;

- Deve-se priorizar a formação de grupos de adolescentes como espaço de valorização e assessoramento para o autocuidado, reforçando os fatores de promoção e proteção. Incluir o adolescente do sexo masculino.

- Deve-se discutir e aperfeiçoar a dinâmica das ações voltadas para 0 adolescente, família e comunidade, por meio de uma abordagem interdisciplinar e intersetorial de forma que se determine um protocolo de atenção ao adolescente, privilegiando as especificidades deste segmento na perspectiva de complementaridade.

Acredita-se que este estudo possa contribuir para o trabalho das enfermeiras na atenção básica, possibilitando reflexões da prática e ressignificação dos conceitos no aprimoramento do cuidar/cuidado na perspectiva da promoção da saúde. Espera-se, também, subsidiar a implementação das políticas de promoção da saúde.

\section{REFERÊNCIAS}

1.Buss PM. Uma reflexão ao conceito de promoção da saúde. In: Czeresnia D, Freitas CM, organizadores. Promoção da saúde: conceitos, reflexões, tendências. Rio de Janeiro: Fiocruz; 2003.

2. Rouquayrol MZ, Goldbaum M. Epidemiologia, história natural da doença. In: Rouquayrol MZ, Almeida Filho N. Epidemiologia e saúde. $6^{a}$ ed. Rio de Janeiro: Medsi; 2003.

3.Ministério da Saúde (BR). As cartas da promoção da saúde. Brasília (DF); 2002. 122 p. (Série: B. Textos Básicos em Saúde).

4Aguiar MIF, Costa LB, Ribeiro MAS, Barroso MGT. Promoção da saúde e doenças sexualmente transmissíveis: a gestante como foco da atenção. Rev RENE. 2004; 5(2): 66-72.

5.Ministério da Saúde (BR). Secretaria de Vigilância em Saúde. Secretaria de Atenção à Saúde. Política Nacional de Promoção da Saúde. Brasília (DF); 2006.60 p. (Série B. Textos Básicos de Saúde)

6.Secretaria de Saúde (CE). Manual das equipes de saúde da família. $2^{\text {a }}$ ed. Fortaleza: SESA; 2004.

7.Gurgel MGI. Prevenção da gravidez na adolescência: atuação da enfermeira na perspectiva da promoção da saúde [dissertação]. Fortaleza: Universidade Federal do Ceará; 2008. 
8.Giovanela L, Mendonça MHM, Almeida PF, Escorel S, Senna MCM, Fausto MCR et al. Saúde da família: limites e possibilidades para uma abordagem integral de atenção primária da saúde no Brasil. Cienc Saude Colet. 2009; 14(3): 783-94.

9.Barroso GT, Vieira NFC, VarelaZMV, organizadores. Educação em saúde no contexto da promoção humana. Fortaleza: Ed Demócrito Rocha; 2003.

10.Gurgel MGI, Alves MDS, Vieira NFC Pinheiro PNC, Barroso GT. Gravidez na adolescência: tendência na produção científica de enfermagem. Esc Anna Nery. 2008; 12(4):800-06.

11.Conselho Regional de Enfermagem (CE). Resolução 240/00. Código de Ética dos Profissionais de Enfermagem. Fortaleza: Pouchain Ramos; 2002.

12.García ML, Gómez BA, Becerril LC, Rojas AM. Cuidados de la saludparadigma del personal de enfermeros en México: la reconstrucción del camino. Esc Anna Nery. 2009; 13(2): 287-96.

13.Ministerio da Saúde (BR). Conselho Nacional de Saúde. Resolução nº 196, de 10 de outubro de 1996. Diretrizes e normas regulamentadoras de pesquisas envolvendo seres humanos. Bioética 1996; 4(2 supl.): 1525.

14.Spink MJ, organizadora. Práticas discursivas e produção de sentido no cotidiano: aproximações teóricas e metodológicas. São Paulo: Cortez; 1999.

15.Fleury-Teixeira P, Vaz FAC, Campos FCC, Álvares J, Aguiar RAT, Oliveira VA. Autonomia como categoria central no conceito de promoção de saúde. Cienc Saude Colet. 2008; 13(supl. 2): 2115-122.

16.Santos FM, David CL. Which empowerment, which health promotion? Conceptual convergences and divergences in preventive health practices. Cad Saude Publica. 2009; 25(1): 68-76. 